\title{
TOPOLOGICALLY DISTINCT LAGRANGIAN AND SYMPLECTIC FILLINGS
}

\author{
Chang Cao, Nathaniel Gallup, Kyle Hayden and Joshua M. Sabloff
}

\begin{abstract}
We construct infinitely many Legendrian links in the standard contact $\mathbb{R}^{3}$ with arbitrarily many topologically distinct Lagrangian fillings. The construction is used to find links in $S^{3}$ that bound topologically distinct pieces of algebraic curves in $B^{4} \subset \mathbb{C}^{2}$, is applied to find contact 3-manifolds with topologically distinct symplectic fillings, and is generalized to higher dimensions.
\end{abstract}

\section{Introduction}

Under certain conditions, the smooth topology of a symplectic manifold is governed by the contact topology of its boundary. The first realization of this principle was Eliashberg's [17] proof that any symplectically aspherical filling of the standard contact $S^{3}$ is diffeomorphic to $B^{4}$; for similar results, see [31,34] in high dimensions and $[30,35,36,45,47]$ in low dimensions. In the relative setting, the original principle was first realized by a theorem of Chantraine [7]: if $L$ is an orientable Lagrangian filling of an oriented Legendrian link in the boundary of a Stein surface, then

$$
t b(\Lambda)=-\chi(L) .
$$

In particular, if $\Lambda$ is connected - i.e., a knot - then the topology of a Lagrangian filling of $\Lambda$ is completely determined by $t b(\Lambda)$.

While recent work has revealed a wide variety of failures of this principle in the case of symplectic manifolds - there are contact 3-manifolds with arbitrarily many non-homeomorphic fillings [28], infinitely many non-homeomorphic fillings [37,38,43], and even infinitely many homeomorphic but not diffeomorphic fillings $[1,2]$ — little is known in the relative setting. In the somewhat softer setting of symplectic fillings of transverse knots in $S^{3}$, Geng [23] notes that Auroux et al. [3] found an example of a transverse braid with two topologically distinct symplectic fillings. Motivated by Chantraine's result and by a question of Boileau and Fourrier [4, Question 1] in an analogous situation in the realm of algebraic curves in $\mathbb{C}^{2}$, we ask:

Question 1.1. Is the topology of a Lagrangian filling of an oriented Legendrian link in $S^{3}$ completely determined by its Thurston-Bennequin invariant?

In contrast to the case of Legendrian knots, the main theorem of this paper implies that the answer to this question is a resounding no. We call a Legendrian link with topologically distinct Lagrangian fillings polyfillable. Let $p(N)$ denote the number of partitions of the natural number $N$, i.e., the number of ways of decomposing $N$ into a sum of positive integers.

Received by the editors August 18, 2013.

2010 Mathematics Subject Classification. 57R17, 53D12. 
Theorem 1.2. For every integer $N>1$, there exists a Legendrian link $\Lambda \subset\left(S^{3}, \xi_{0}\right)$ with $p(N)$ non-homeomorphic exact orientable Lagrangian fillings.

In fact, we can construct links that have an enormous amount of flexibility in their polyfillability, and the theorem above is a simple corollary of one of several more refined theorems that we state and prove in Section 3.

Though the theorem above is interesting in and of itself, we also apply it in three different directions. First, Theorem 1.2 leads to a solution of Boileau and Fourrier's original question:

Theorem 1.3. For every integer $N>1$, there exists a link $K \subset S^{3}$ with $p(N)$ non-singular complex algebraic curves that intersect $S^{3}$ transversally in $K$ with the property that the intersections of the curves with $B^{4}$ are pairwise non-homeomorphic.

We note that the method by which we connect Theorems 1.2 and 1.3 shows that Auroux et al.'s [3] pair of transverse fillings also yields a negative answer to Boileau and Fourrier's question.

Second, Theorem 1.2 may also be applied to the construction of multiple strong symplectic fillings of contact manifolds. We will show below that we may perturb the Lagrangian fillings from Theorem 1.2 into symplectic fillings of transverse links. Taking double branched covers over the symplectic fillings then yields the following:

Theorem 1.4. For every positive integer $N$, there exists a contact 3 -manifold $(Y, \alpha)$ with strong symplectic fillings $\left(X_{1}, \omega_{1}\right), \ldots,\left(X_{N}, \omega_{N}\right)$ that have the same Euler characteristic, but for which

$$
\operatorname{rank} H_{3}\left(X_{k}, Y\right)=k-1 \text {. }
$$

In particular, the fillings $X_{k}$ do not differ solely by a symplectic blow-up.

We note that this result is not as spectacular as some of those mentioned in the discussion of symplectic fillings, above, but we believe that the branched cover technique is novel.

Finally, we may extend Theorem 1.2 to higher dimensions while dropping the requirement that the Legendrian is disconnected:

Theorem 1.5. For all integers $n ; N>1$, there exists a connected Legendrian submanifold $\Lambda \subset\left(S^{2 n+1}, \xi_{0}\right)$ with $N$ non-homeomorphic connected exact orientable Lagrangian fillings.

As we shall discuss in Section 4.4, this construction implies that there are Legendrian submanifolds with arbitrarily large sets of generating family homology or linearized Legendrian contact homology invariants, generalizing the result of Melvin and Shrestha [32] for knots in the standard contact $\mathbb{R}^{3}$.

The remainder of the paper is organized as follows. We sketch the relevant background material on Lagrangian and symplectic fillings in Section 2. The proof of Theorem 1.2 appears in Section 3, while its application to the proofs of Theorems 1.3-1.5 appears in Section 4. 


\section{Lagrangian and symplectic fillings}

We begin this section by setting down some of the basic language of Legendrian knot theory, Lagrangian fillings, and symplectic fillings of contact manifolds. We assume a basic familiarity with Legendrian knot theory and contact topology; for further background, see the introductory articles of Etnyre [19,20] or Geiges' [22] text. With the appropriate language established, we review the geometric tools for constructing Lagrangian surfaces that underlie the main theorem.

2.1. Lagrangian and symplectic fillings. We begin by considering Legendrian links in a contact manifold $(Y, \alpha)$ and their Lagrangian fillings in the symplectization $\left(\mathbb{R} \times Y, d\left(e^{t} \alpha\right)\right)$. A Lagrangian submanifold $L \subset \mathbb{R} \times Y$ is a Lagrangian cobordism between Legendrian links $\Lambda_{-}, \Lambda_{+} \subset Y$ if there exist real numbers $T_{-}<T_{+}$so that

$$
\begin{gathered}
L \cap\left(-\infty, T_{-}\right] \times Y=\left(-\infty, T_{-}\right] \times \Lambda_{-}, \\
L \cap\left[T_{+}, \infty\right) \times Y=\left[T_{+}, \infty\right) \times \Lambda_{+} .
\end{gathered}
$$

We write $\Lambda_{-} \prec_{L} \Lambda_{+}$to denote this situation. In the language of [8], this definition requires our Lagrangian cobordisms to be collared. A Lagrangian cobordism from $\emptyset$ to $\Lambda$ is a Lagrangian filling of $\Lambda$. In this paper, we will be primarily concerned with the standard contact structures on $\mathbb{R}^{3}$ and $S^{3}$.

A symplectic manifold $(X, \omega)$ is a strong symplectic filling of a contact manifold $(Y, \alpha)$ if $Y=\partial X$ and there exists a non-vanishing Liouville vector field $W$ for $\omega$ that is transverse to $Y$ and so that $\alpha=\left.\iota_{W} \omega\right|_{Y}$. In lieu of the Liouville vector field, we could also have specified that there is a collar neighborhood of $Y$ in $X$ that is symplectomorphic to $\left((-a, 0] \times Y, d\left(e^{t} \alpha\right)\right)$. The canonical example of a strong symplectic filling is the filling of the standard contact structure on $S^{3}$ by the standard symplectic structure on $B^{4}$. Notice that just as any Legendrian link $\Lambda$ in the standard contact $\mathbb{R}^{3}$ can be thought of as living in the standard contact $S^{3}$, so any Lagrangian filling of $\Lambda$ immediately yields a properly embedded collared Lagrangian surface in $B^{4}$. We may thus make our constructions in the symplectization of $\mathbb{R}^{3}$ but state the resulting theorems in the filling $B^{4}$ of $S^{3}$.

2.2. Constructions of Lagrangian fillings. Our main geometric tool for constructing Lagrangian fillings, embodied by the theorem below, was first announced by Ekholm et al. [15]. The first part of the theorem was proven by Chantraine [7], and the last two parts are formulated as in [6]; see also Dimitroglou Rizell's [11] work.

Theorem 2.1 ([6,7,11,15]). If two oriented Legendrian links $\Lambda_{-}$and $\Lambda_{+}$in the standard contact $\mathbb{R}^{3}$ are related by any of the following three moves, then there exists an oriented exact Lagrangian cobordism $\Lambda_{-} \prec_{L} \Lambda_{+}$.

Isotopy: $\Lambda_{-}$and $\Lambda_{+}$are Legendrian isotopic.

0-handle: The front of $\Lambda_{+}$is the same as that of $\Lambda_{-}$except for the addition of a disjoint Legendrian unknot as in the top of Figure 1.

1-handle: The fronts of $\Lambda_{ \pm}$are related as in the bottom of Figure 1.

Example 2.2. Let $T_{n}$ denote the Legendrian $(2, n)$-torus link, where $n>0$ denotes the number of positive half-twists, shown in Figure 2. 


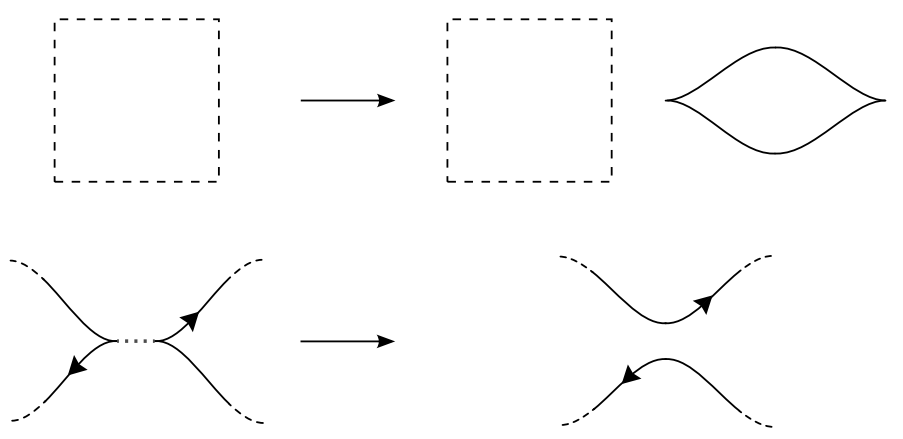

FiguRE 1. The second and third moves of Theorem 2.1.

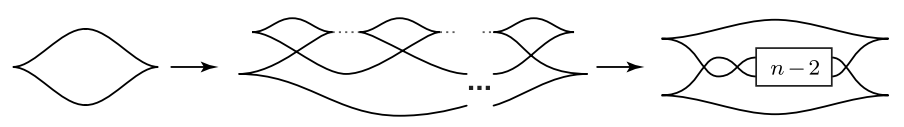

Figure 2. Construction of a filling of a $(2, n)$-torus link.

The link $T_{n}$ admits a Lagrangian filling with Euler characteristic $2-n$. To see this, first create an unknot and then use the first Reidemeister move $n$ times on the top of the unknot as in Figure 2. Add $n-1$ 1-handles to obtain $T_{n}$. A simple calculation shows that $t b\left(T_{n}\right)=n-2$, so by equation (1.1), the Euler characteristic of the filling is $2-n$.

In Section 4.4, we will require Golovko's [24] cobordism spinning construction and a generalization of 1-handle attachments to higher dimensions. These constructions will be discussed as they are needed.

From now on, we will deliberately confuse the notation for a Legendrian link and its front projection. In light of the above theorem, we say that an oriented Legendrian link has a decomposable Lagrangian filling if there exists a sequence of moves from Theorem 2.1 which takes $\emptyset$ to a front diagram of the link. The Lagrangian fillings constructed in this paper are decomposable, but this is not the case for all Lagrangian fillings, as Chaintraine proved in [9].

Remark 2.3. The orientations of the components of a link are important in the definition above. For example, only one of the two orientations we may assign to the Hopf link allows for a decomposable filling.

\section{Polyfillable Legendrian links}

In this section, we prove Theorem 1.2. As mentioned in the introduction, we actually prove several more refined theorems that show that we can finely control the distribution of the topology of a filling across the components of a Legendrian link.

3.1. A first example. We begin our discussion with a simple example of a polyfillable link $\Lambda^{*}$, which appears on the far right of Figure 3. Note that this example is already enough to answer Question 1.1. Constructions of two topologically distinct fillings of $\Lambda^{*}$ may be described as follows: 


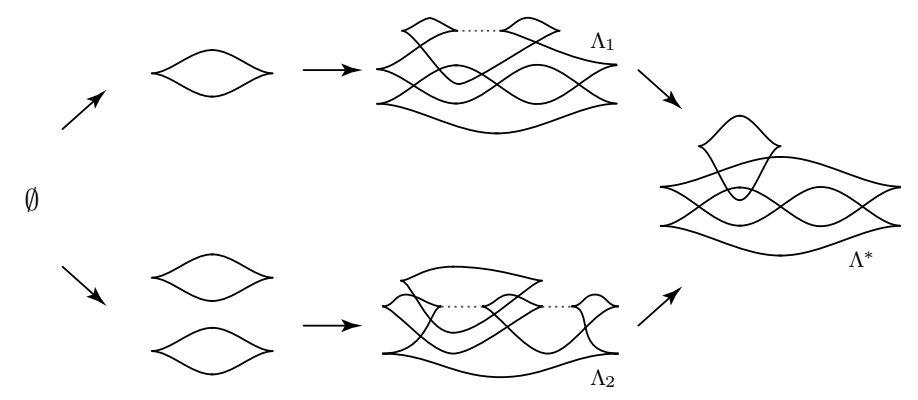

FiguRE 3. The link $\Lambda^{*}$ on the right has two topologically distinct Lagranian fillings: the top filling is an annulus, while the bottom is the union of a disk and a punctured torus.

Construction A: Create a 0 -handle and obtain $\Lambda_{1}$ via Legendrian isotopy. Attach a 1-handle to obtain $\Lambda^{*}$. Notice that since there is but one 0 -handle, the filling created is a connected surface - in particular, it is an annulus.

Construction B: Create two 0-handles and obtain $\Lambda_{2}$ via Legendrian isotopy. Then, add two 1-handles to obtain $\Lambda^{*}$. Since no 1-handle is attached between the two link components, the filling created is a disconnected surface; the top component of $\Lambda^{*}$ bounds a disk, while the bottom bounds a punctured torus.

3.2. The tangle replacement construction. The polyfillable link $\Lambda^{*}$ discussed above may be viewed as an entanglement of the unknot and the trefoil. The manner in which these link components are entangled may be generalized through the following tangle replacement procedure.

Definition 3.1. If a Legendrian link $\Lambda$ contains the tangle in the center of Figure 4 with the top two strands belonging to a component $\Lambda_{0}$ and the bottom three strands belonging to a sublink $\Lambda_{1}$ that does not contain $\Lambda_{0}$, then we say that $\Lambda$ contains a $\Pi$ tangle. Denote the tangle replacements on the left and right sides of Figure 4 by $\Lambda_{\Pi}^{-}$and $\Lambda_{\Pi}^{+}$, respectively.

The top and bottom sequences in Figure 5 define topologically distinct cobordisms between $\Lambda_{\Pi}^{-}$and $\Lambda_{\Pi}^{+}$. In the upper sequence, the join construction, $\Lambda_{0}^{-}$and $\Lambda_{1}^{-}$are merged by attaching a Lagrangian 1-handle. It follows that $\Lambda_{0}^{+}$and $\Lambda_{1}^{+}$are contained
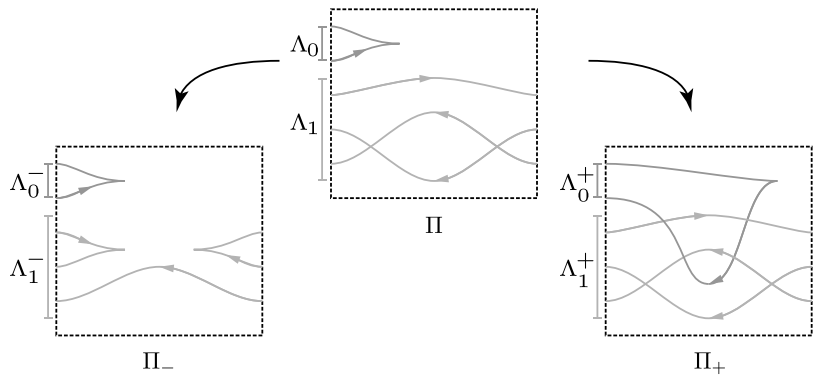

FiguRE 4 . The tangles $\Pi, \Pi_{-}$and $\Pi_{+}$. 


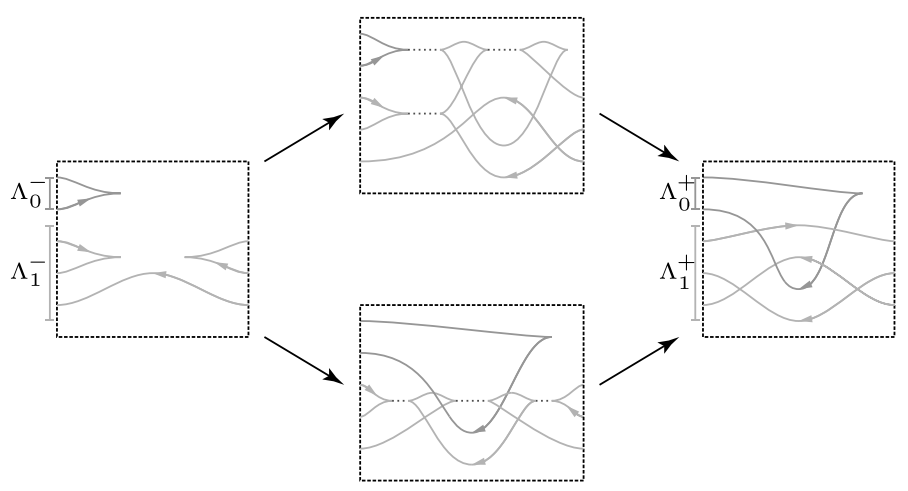

Figure 5. The upper sequence defines the join construction and the lower sequence defines the split construction. In each case, Legendrian isotopy is implied between the first and second diagrams, and dotted horizontal bars indicate 1-handle attachments.

in the boundary of a single connected component of the cobordism. In the lower sequence, the split construction, all 1-handles are attached within $\Lambda_{1}^{-}$. Thus, $\Lambda_{0}^{+}$ and $\Lambda_{1}^{+}$belong to the boundaries of disjoint components.

The following theorem recasts the above discussion in a computationally useful form, using equation (1.1) to compute the changes in the Euler characteristics of the fillings.

Theorem 3.2. Suppose the Legendrian link $\Lambda$ contains a $\Pi$ tangle involving the sublinks $\Lambda_{0}$ and $\Lambda_{1}$. If $\Lambda_{\Pi}^{-}$has a Lagrangian filling $L^{-}$with $\Lambda_{0}^{-}$and $\Lambda_{1}^{-}$lying on the boundaries of different connected components $L_{0}^{-}$and $L_{1}^{-}$of $L^{-}$, then $\Lambda_{\Pi}^{+}$has two topologically distinct fillings characterized as follows:

(1) The join construction yields a filling $L^{+}$of $\Lambda_{\Pi}^{+}$with one fewer connected component than $L^{-}$. The Euler characteristic of the component arising from the merging of two previously disconnected components is $\chi\left(L_{0}^{-}\right)+\chi\left(L_{1}^{-}\right)-3$.

(2) The split construction yields a filling $L^{+}$of $\Lambda_{\Pi}^{+}$with the same number of connected components as $L^{-}$. The component of $L^{+}$extending $L_{0}^{-}$has the same Euler characteristic as $L_{0}^{-}$, while the component that extends $L_{1}^{-}$has Euler characteristic $\chi\left(L_{1}^{-}\right)-3$.

3.3. An infinite family of polyfillable links. We are now ready to construct a family of polyfillable links; Theorem 1.2 will be a direct consequence. The family will be indexed by compositions of an integer $N$, i.e., positive integer vectors $X=$ $\left(x_{1}, \ldots, x_{n}\right)$ such that $\sum_{i=1}^{n} x_{i}=N$. We say that $n$ is the length of $X$. Following [44, p. 18], the set of compositions $C(N)$ of $N$ is in bijective correspondence with the power set of $\{1, \ldots, N-1\}$, which can be seen as follows: represent $N$ as a line of $N$ dots and represent a length $k$ composition of $N$ as a set of $k-1$ vertical bars that separate the dots into sets whose sizes are determined by the composition. For example, the composition $(2,1,3)$ of 6 looks like the following: 
Thus, the set $C(N)$ inherits a natural partial order $\leq$ from the partial order on the power set given by inclusion. From the perspective of dot-and-bar diagrams, we have $Y \leq X$ if the dot-and-bar diagram of $Y$ can be obtained from that of $X$ by removing zero or more bars. Numerically, $Y$ is the result of adding together disjoint strings of adjacent entries in $X$. Note that taking the set of compositions of $N$ modulo permutation of the components yields the set of partitions of $N$.

With this notation in hand, we may describe the family of polyfillable links and their fillings.

Theorem 3.3. Given a composition $X$ of an integer $N>1$, where $X$ has length $n>$ 1 , there exists a polyfillable Legendrian link $\Lambda_{X}$ such that for any composition $Y \leq X$, there exists a Lagrangian filling $L_{Y}$ whose ith component has Euler characteristic $-y_{i}$.

Proof. Consider the split $\operatorname{link} \Lambda^{\mathrm{s}}=\bigsqcup_{i=1}^{n} \Lambda_{i}$ depicted in Figure 6, where $\Lambda_{i}$ is a $\left(2, x_{i}+2\right)$-torus link. Notice that the bottom-right cusp of $\Lambda_{i}$ and the two rightmost crossings of $\Lambda_{i+1}$ form a $\Pi$ tangle, giving rise to the $n-1 \Pi$ tangles shown in Figure 6 .

To create $\Lambda_{X}$, simply replace these $n-1 \Pi$ tangles by $\Pi_{+}$. To understand the fillings $L_{Y}$, suppose that $Y \leq X$; i.e., suppose that

$$
Y=\left(x_{1}+\cdots+x_{i_{1}}, x_{i_{1}+1}+\cdots+x_{i_{2}}, \ldots, x_{i_{k}+1}+\cdots+x_{n}\right) .
$$

To create $L_{Y}$, first replace the aforementioned $\Pi$ tangles of $\Lambda^{s}$ by $\Pi_{-}$. The resulting link is a split link whose components are unknots and $(2, m)$-torus knots, all of which are individually fillable by Example 2.2. Complete $L_{Y}$ by performing the split construction on the $\Pi$ tangles between the sublinks $\Lambda_{i_{j}}$ and $\Lambda_{i_{j}+1}, j=1, \ldots, k$, and the join construction on the $\Pi$ tangles between the other sublinks.

To verify that we have, indeed, constructed the filling $L_{Y}$ described in the statement of the theorem, first note that Theorem 3.2, used inductively, tells us that the resulting filling has $k+1$ components. Further, the Euler characteristic of the $j$ th component may be computed using equation (1.1), which equates the Euler characteristic of each component to the negative of the Thurston-Bennequin invariant of its boundary. Since the boundary of the $j$ th component consists of $\left(2, x_{j}+2\right)$-torus knots linked together, and since one may easily see that the crossings between two knots contribute zero
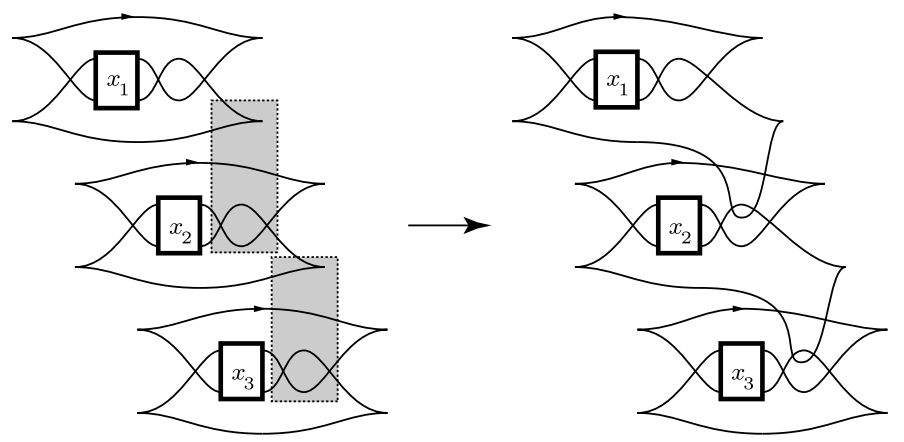

FiguRE 6 . The split link $\Lambda^{\mathrm{s}}$ corresponding to a length-3 composition and its conversion to $\Lambda_{X}$ via several $\Pi$ tangle replacements. 
to the writhe of the diagram, we may conclude that the Euler characteristic of the $(j+1)^{\text {st }}$ component of $L_{Y}$ is $-\sum_{m=i_{j}+1}^{i_{j+1}} x_{m}$, as required, where we let $i_{0}=0$.

To derive Theorem 1.2 from 3.3 , simply consider the composition $X=(1,1, \ldots, 1)$ of $N$. Note that the link $\Lambda_{X}$ in this case is necessarily composed of trefoil components. The composition $X$ is maximal with respect to the partial order, and hence the number of topologically distinct fillings of $\Lambda_{X}$ is equal to the number of partitions of $N$, as the topological type of the filling is insensitive to order.

Looking at the increasing sequence

$$
(N) \leq(1, N-1) \leq(1,1, N-2) \leq \cdots \leq(1,1, \ldots, 1)
$$

of compositions of $N$, we obtain the following corollary:

Corollary 3.4. For every positive integer $N$, there exists an $N$-component Legendrian link $\Lambda_{N} \subset\left(S^{3}, \xi_{0}\right)$ such that for any $1 \leq k \leq N$, there exists a Lagrangian filling $L_{k, N} \subset\left(B^{4}, \omega_{0}\right)$ of $\Lambda_{N}$ with $k$ connected components.

\section{Applications of Theorem 1.2}

The goal of this section is to derive Theorems 1.3-1.5 from Theorem 1.2.

4.1. Symplectic fillings of transverse links. The first step in the proofs of Theorems 1.3 and 1.4 is to $C^{\infty}$ approximate a Lagrangian filling of a Legendrian link by a symplectic filling of a transverse link.

Lemma 4.1. Let $(X, L, \omega)$ be an oriented strong Lagrangian filling of $(Y, \Lambda, \alpha)$. The Lagrangian surface $L$ may be $C^{\infty}$ approximated by a symplectic surface $L^{+}$that satisfies:

(1) $\left.\omega\right|_{L^{+}}>0$ and

(2) $\partial L^{+}$is a positive transverse link smoothly isotopic to $\Lambda$.

Proof. By Lemma 2.3.A in Eliashberg's [18] paper, it suffices to perturb $L$ in a collar neighborhood of $\partial L$ so that the boundary of the resulting surface is a transverse link and so that $\omega$ is positive on a neighborhood of the boundary of the perturbed surface.

We work in local coordinates in a collar neighborhood $(0,1] \times S^{1} \times \mathbb{R}^{2}$ of the boundary of $L$, where the coordinates are $(s, \theta, x, y)$ and the symplectic form is $\omega=$ $d\left(e^{\mathrm{s}}(\cos \theta d x-\sin \theta d y)\right)$. The Lagrangian $L$ is the image of the embedding

$$
\begin{aligned}
\phi:(0,1] \times S^{1} & \rightarrow(0,1] \times S^{1} \times \mathbb{R}^{2} \\
(s, t) & \mapsto(s, t, 0,0) .
\end{aligned}
$$

Orient $L$ by specifying that $(0,1] \times S^{1}$ is oriented by $d s \wedge d t$.

To perturb $L$, we choose a smooth increasing function $\sigma:(0,1] \rightarrow \mathbb{R}$ that is equal to 0 near 0 and is equal to $\epsilon>0$ near 1 . Define the perturbed surface using the embedding $\phi_{+}$given by the following formula:

$$
\phi_{+}(s, t)=(s, t, \sigma(s) \sin t, \sigma(s) \cos t) .
$$

It is clear from the definition of $\sigma$ that this perturbation may be made as small as desired and can be extended to the identity outside of the given neighborhood, so it 
suffices to show that $\phi_{+}^{*} \omega$ is a non-negative multiple of $d s \wedge d t$. This is a straightforward computation:

$$
\phi_{+}^{*} \omega=e^{\mathrm{s}}\left(\sigma(s)+\sigma^{\prime}(s)\right) d s \wedge d t .
$$

Since $e^{\mathrm{s}}\left(\sigma(s)+\sigma^{\prime}(s)\right) \geq 0$, the conclusion follows.

4.2. Algebraically polyfillable links. In this subsection, we prove Theorem 1.3, namely that for every integer $N>1$, there exists a link $K \subset S^{3}$ with $p(N)$ nonsingular complex algebraic curves that intersect $S^{3}$ transversally in $K$ with the property that the intersections of the curves with $B^{4}$ are pairwise non-homeomorphic. Let $\Lambda$ be the Legendrian link constructed for Theorem 1.2 and let $L_{Y}$ be the Lagrangian filling corresponding to the composition $Y$ of $N$. By Lemma 4.1, we may perturb $L_{Y}$ to a symplectic surface $L_{Y}^{+}$in $B^{4}$ whose boundary is a positive transverse link $K$. By Theorem 2 of [5], the surface $L_{Y}^{+}$is quasi-positive. Finally, Rudolph [39] proved (as interpreted in [40, Section 4]) that every quasi-positive surface $S$ is isotopic to a smooth piece of an algebraic curve inside $B^{4}$ that intersects $S^{3}$ transversally in a link isotopic to $\partial S$. This completes the proof.

4.3. Polyfillable contact 3 -manifolds. We now pivot from fillings of links to fillings of contact 3 -manifolds.

Fix $N>1$ and let $L_{1}, \ldots, L_{N}$ be the Lagrangian fillings of the Legendrian link $\Lambda_{N}$ provided by Corollary 3.4. We will abuse notation and assume that the surfaces $L_{k}$ have already been perturbed, as in Lemma 4.1, to symplectic surfaces with transverse boundary. Define $\left(X_{k}, \omega_{k}\right)$ to be the double branched cover of $B^{4}$, branched over $L_{k}$, and let $(Y, \alpha)$ be the double branched cover of $S^{3}$, branched over $\Lambda_{N}$. The construction of the symplectic form on the double branched cover may be found in [25], for example. Since we know that the boundaries of the symplectic surfaces $L_{k}$ have collar neighborhoods and that $B^{4}$ has a collar neighborhood symplectomorphic to a portion of the symplectization of $S^{3}$, we know that the same is true for $X_{k}$ and $Y$. It follows that $\left(X_{k}, \omega_{k}\right)$ is a strong filling of $(Y, \alpha)$. Alternatively, as pointed out by Loi and Piergallini [29, Theorem 3], the work in the previous subsection actually allows us to construct an analytic double branched cover over an algebraic curve; in this case, we see that $\left(X_{k}, \omega_{k}\right)$ is, in fact, a Stein filling.

To finish the proof of Theorem 1.4, then, we need only prove the following lemma:

Lemma 4.2. The rank of $H_{3}\left(X_{k}, Y\right)$ is $k-1$, but all $X_{k}$ have the same Euler characteristic.

Proof. The key tool in this proof is the following long exact sequence, adapted from $\left[27\right.$, Theorem 1]: ${ }^{1}$

$$
\cdots \rightarrow H_{*}\left(B^{4}, L_{k} \cup S^{3}\right) \rightarrow H_{*}\left(X_{k}, Y\right) \rightarrow H_{*}\left(B^{4}, S^{3}\right) \rightarrow \cdots .
$$

The long exact sequence shows that the Euler characteristic $\chi\left(X_{k}, Y\right)$ may be computed from the Euler characteristics $\chi\left(B^{4}, S^{3}\right)$ (which is simply 1) and $\chi\left(B^{4}, L_{k} \cup\right.$ $\left.S^{3}\right)$. To compute the latter quantity, we use the long exact sequence of the triple

\footnotetext{
${ }^{1}$ While the long exact sequence in [27] is only discussed for $\mathbb{Z}_{2}$ coefficients, it is clear that if both the base and the branched cover are orientable, as they are here, the proof carries over to $\mathbb{Z}$ coefficients.
} 
$\left(B^{4}, L_{k} \cup S^{3}, S^{3}\right)$, which shows that $\chi\left(B^{4}, L_{k} \cup S^{3}\right)$ depends only on $\chi\left(L_{k} \cup S^{3}, S^{3}\right)$. Excision shows that

$$
\chi\left(L_{k} \cup S^{3}, S^{3}\right)=\chi\left(L_{k}, \Lambda_{N}\right),
$$

which does not depend on $k$ by Chantraine's theorem (1.1). Thus, $\chi\left(X_{k}, Y\right)$ does not depend on $k$.

On the other hand, the rank of $H_{3}\left(X_{k}, Y\right)$ does depend on $k$. Theorem 2 of [27] implies that the connecting homomorphism

$$
\partial_{*}: H_{4}\left(B^{4}, S^{3}\right) \rightarrow H_{3}\left(B^{4}, L_{k} \cup S^{3}\right)
$$

is injective, and hence it suffices to compute $H_{3}\left(B^{4}, L_{k} \cup S^{3}\right)$. Since $H_{3}\left(B^{4}, S^{3}\right)$ and $H_{2}\left(B^{4}, S^{3}\right)$ obviously vanish, the long exact sequence of the triple $\left(B^{4}, L_{k} \cup S^{3}, S^{3}\right)$ and excision yield the isomorphisms

$$
H_{3}\left(B^{4}, L_{k} \cup S^{3}\right) \simeq H_{2}\left(L_{k} \cup S^{3}, S^{3}\right) \simeq H_{2}\left(L_{k}, \Lambda_{N}\right) .
$$

Since $L_{k}$ has $k$ components, the rank of $H_{2}\left(L_{k}, \Lambda_{N}\right)$ is $k$. The lemma now follows from (4.1) and (4.2).

4.4. Higher-dimensional fillings. We finish our exploration of the applications of Theorem 1.2 by examining its generalization to higher dimensions and the consequences of the generalization for the generating family homology and linearized Legendrian contact homology invariants.

4.4.1. Connected polyfillable Legendrian submanifolds. To generalize Theorem 1.2 to higher dimensions, we need two further constructions of Lagrangian cobordisms. The first is spinning. Suppose that a front diagram for a Legendrian submanifold $\Lambda \subset$ $\mathbb{R}^{2 n+1}$ lies in the half-space $x_{1}>0$. The front spinning construction of Ekholm et al. [14] produces a new Legendrian submanifold $\Sigma \Lambda \subset \mathbb{R}^{2 n+3}$ that is homeomorphic to $\Lambda \times$ $S^{1}$. Golovko [24] extended this construction to Lagrangian cobordisms: a Lagrangian cobordism $\Lambda_{-} \prec_{L} \Lambda_{+}$lying in the $x_{1}>0$ half-space may be spun to a Lagrangian cobordism $\Sigma \Lambda_{-} \prec_{\Sigma L} \Sigma \Lambda_{+}$.

The second construction is a generalization of the 1-handle attachment in Theorem 2.1 to Legendrian surfaces in $\mathbb{R}^{5}$. As formulated in [6], but see also [11], if two Legendrian surfaces $\Lambda_{-}$and $\Lambda_{+}$in the standard contact $\mathbb{R}^{5}$ are related as in Figure 7 , then there is an oriented Lagrangian cobordism $\Lambda_{-} \prec_{L} \Lambda_{+}$. The cobordism consists of attaching a Lagrangian 1-handle to the negative end of a cobordism, $(-\infty,-T] \times \Lambda_{-} \subset \mathbb{R} \times \mathbb{R}^{5}$.

We now have the tools to prove Theorem 1.5.

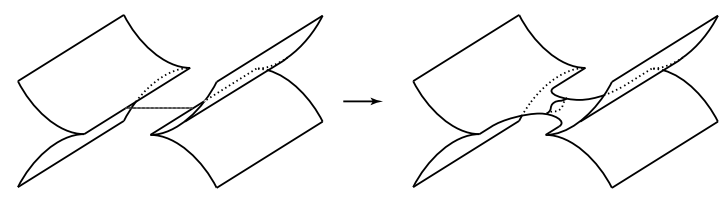

FiguRE 7. Attaching a Lagrangian 1-handle to a Legendrian surface $\Lambda_{-}$. The resulting Lagrangian cobordism is orientable if the orientations of $\Lambda_{-}$are opposite on either side of the dotted line 
Proof of Theorem 1.5. We begin with the link $\Lambda_{N}$ of Corollary 3.4, whose components are all trefoil knots. Note that $t b\left(\Lambda_{N}\right)=N$. We make a slight modification to $\Lambda_{N}$ using one Reidemeister I move for all but the bottom-most component; see Figure 8. By Theorem 2.1, this modification does not change the fillability properties of the link. If a filling $L_{k}$ of $\Lambda_{N}$ has $k$ connected components, then equation (1.1) implies that

$$
\operatorname{rank} H_{1}\left(L_{k}\right)=N+k \text {. }
$$

Spin $\Lambda_{N}$ to a Legendrian surface $\Sigma \Lambda_{N}$ in $\mathbb{R}^{5}$, and spin the fillings $L_{k}$ to fillings $\Sigma L_{k}$ of $\Sigma \Lambda_{N}$. The Künneth theorem and equation (4.3) imply that

$$
\operatorname{rank} H_{1}\left(\Sigma L_{k}\right)=N+2 k \text {. }
$$

Finally, attach $N-1$ Lagrangian 1-handles at the positions indicated in Figure 8 to obtain Lagrangian fillings $\Sigma^{\prime} L_{k}$ of a new Legendrian surface $\Lambda_{N}^{\prime}$. The resulting Legendrian is connected, as are all of the resulting Lagrangian fillings. The fillings are still topologically distinct, however, as we may compute

$$
\operatorname{rank} H_{1}\left(\Sigma^{\prime} L_{k}\right)=2 k+1 \text {. }
$$

Finally, we may repeatedly spin the resulting Legendrian and its fillings to extend the result to Legendrian submanifolds of arbitrarily high dimension.

Remark 4.3. The Legendrian submanifolds constructed in the proof above are the product of a genus $N$ surface with an $(n-2)$-dimensional torus. It is possible to use either a refinement of the spinning construction for fronts and cobordisms with boundary in [6] or a sequence of higher embedded surgeries as in $[6,11]$ to produce polyfillable Legendrian spheres of dimension $n>1$.

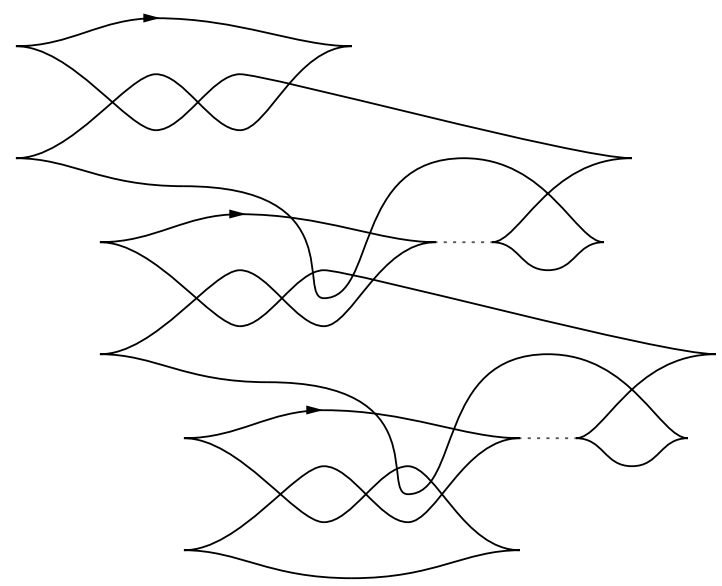

FIGURE 8. Spinning the pictured link and then performing 1-handle attachments at the dotted lines yields a connected polyfillable Legendrian surface. 
4.4.2. Chekanov numbers in higher dimensions. Theorem 1.5 has implications for the structure of non-classical Legendrian invariants. In this subsection only, we assume familiarity with generating family invariants of Legendrian submanifolds, as explained in $[21,26,41,46]$, and with Legendrian contact homology and its linearizations, as explained in $[10,14]$. The linearized contact homology invariant is a collection of graded (homology) groups parametrized by augmentations of the Legendrian contact homology differential graded algebra. Similarly, the generating family invariant of a Legendrian $\Lambda$ takes the form of a collection of graded (homology) groups, parametrized by equivalence classes of generating families for $\Lambda$. The cardinalities of these sets are, themselves, Legendrian invariants: the cardinality of the set of linearized Legendrian contact homology groups is called the Chekanov number, while the cardinality of the set of generating family homology groups is called the GF number. These numbers are known to be equal for links in $\mathbb{R}^{3}[21]$ and are conjecturally equal in higher dimensions.

An early question in the theory of Legendrian contact homology, proposed by $\mathrm{Ng}$ [33] and resolved for Legendrian knots in $\mathbb{R}^{3}$ by Melvin and Shrestha [32] (see also Sivek [42]), was whether the Chekanov number could ever be greater than 1 . For Legendrian knots in $\mathbb{R}^{3}$, the answer is yes; in fact, the Chekanov number can be arbitrarily high.

Theorem 1.5 may be used to produce examples of Legendrian submanifolds of $\mathbb{R}^{2 n+1}$ with arbitrarily high Chekanov/GF numbers.

Proposition 4.4. For any integers $n>1$ and $N>0$, there exists a connected Legendrian submanifold $\Lambda \subset \mathbb{R}^{2 n+1}$ with Chekanov/GF number at least $N$.

Proof. We first prove the proposition for the GF number. The proof relies on the fact, proved in [41], that if a Lagrangian filling $L$ of a Legendrian submanifold $\Lambda$ is "compatible" with a generating family $f$ of $\Lambda$, then

$$
G H^{*}(f) \simeq H^{*+1}(L, \Lambda) .
$$

Thus, if the Legendrian $\Lambda$ has $N$ compatible Lagrangian fillings, all of which have different relative cohomology, then the GF number of $\Lambda$ is at least $N$.

It suffices to prove, then, that the Lagrangian fillings $L_{k}$ constructed in the previous subsection are all compatible with a generating family of $\Lambda$. This, however, is a straightforward affair given the results of [6]: the isotopy, handle attachment, and spinning constructions discussed in Theorem 2.1 and Section 4.4.2 all produce compatible Lagrangian cobordisms, so long as the 1-handle attachments join cusps that represent births and deaths of fiber critical points of the generating family with the same Morse index; this is easy to arrange.

The proof of the proposition for the Chekanov number follows the same argument, with equation (4.6) replaced by a similar result that was stated and outlined by Ekholm [13] and proven by Dimitroglou Rizell [12].

Remark 4.5. Examples that prove the proposition above could also be constructed by spinning the examples of Melvin-Shrestha or of Sivek and using the techniques of 
Ekholm and Kálmán [16] to compute the Legendrian contact homology. On the other hand, for each of Melvin-Shrestha's or Sivek's examples, only one of the linearized contact or generating family homologies arises from a filling (as in equation (4.6)), whereas all of the linearized contact or generating family homologies constructed in the proof above arise from Lagrangian fillings.

\section{Acknowledgments}

We thank Matt Hedden for bringing Boileau and Fourrier's question to our attention and for explaining the method for deriving Theorem 1.3 from 1.2. We thank Curtis Greene for explaining the proper viewpoint on compositions and partial orders that we use in stating Theorem 3.3. Finally, we gratefully acknowledge support from NSF grant DMS-0909273.

\section{References}

[1] A. Akhmedov, J. Etnyre, T. Mark and I. Smith, A note on Stein fillings of contact manifolds, Math. Res. Lett. 15(6) (2008), 1127-1132.

[2] A. Akhmedov and B. Ozbagci, Exotic Stein fillings with arbitrary fundamental group, preprint, 2012, available as arXiv: 1212.1743.

[3] D. Auroux, V.S. Kulikov and V. Shevchishin, Regular homotopy of Hurwitz curves, Izv. Ross. Akad. Nauk Ser. Mat. 68(3) (2004), 91-114.

[4] M. Boileau and L. Fourrier, Knot theory and plane algebraic curves, Chaos Solitons Fractals $\mathbf{9}(4-5)$ (1998), 779-792, Knot theory and its applications.

[5] M. Boileau and S. Orevkov, Quasi-positivité d'une courbe analytique dans une boule pseudoconvexe, C. R. Acad. Sci. Paris Sér. I Math. 332(9) (2001), 825-830.

[6] F. Bourgeois, J. Sabloff and L. Traynor, Lagrangian cobordisms via generating families: constructions and geography, in preparation.

[7] B. Chantraine, On Lagrangian concordance of Legendrian knots, Algebr. Geom. Topol. 10 (2010), 63-85.

[8] B. Chantraine, Some non-collarable slices of Lagrangian surfaces, preprint, 2011, available as arXiv: 1108.2969v2.

[9] B. Chantraine, A note on exact Lagrangian cobordisms with disconnected Legendrian ends, preprint, 2013, available as arXiv:1301.6903.

[10] Y. Chekanov, Differential algebra of Legendrian links, Invent. Math. 150 (2002), 441-483.

[11] G. Dimitroglou Rizell, Legendrian ambient surgery and Legendrian contact homology, preprint, 2012, available as arXiv:1205.5544v1.

[12] G. Dimitroglou Rizell, Lifting pseudo-holomorphic polygons to the symplectisation of $P \times R$ and applications, preprint, 2013, available as arXiv:1305.1572.

[13] T. Ekholm, Rational SFT, linearized Legendrian contact homology, and Lagrangian Floer cohomology, in 'Perspectives in analysis, geometry, and topology', (I. Itenberg, B. Jöricke, M. Passare, eds.) 109-145, Progr. Math., 296, Birkhäuser/Springer, New York, 2012.

[14] T. Ekholm, J. Etnyre and M. Sullivan, Non-isotopic Legendrian submanifolds in $\mathbb{R}^{2 n+1}$, J. Differential Geom. 71(1) (2005), 85-128.

[15] T. Ekholm, K. Honda and T. Kálmán, Legendrian knots and exact Lagrangian cobordisms, preprint, 2012, available as arXiv:1212.1519.

[16] T. Ekholm and T. Kálmán, Isotopies of Legendrian 1-knots and Legendrian 2-tori, J. Symplectic Geom. 6(4) (2008), 407-460.

[17] Y. Eliashberg, Filling by holomorphic discs and its applications, in 'Geometry of low-dimensional manifolds, 2 (Durham, 1989)', (S. K. Donaldson, C. B. Thomas, eds.) 45-67, 296, Cambridge University Press, Cambridge, 1990.

[18] Y. Eliashberg, Topology of 2-knots in $\mathbf{R}^{4}$ and symplectic geometry, in 'The Floer memorial volume', (H. Hofer, C. H. Taubes, A. Weinstein, E. Zehnder, eds.) 335-353, Progr. Math., 133, Birkhäuser, Basel, 1995. 
[19] J. Etnyre, Introductory lectures on contact geometry, in 'Topology and geometry of manifolds (Athens, GA, 2001)', (G. Matić, C. McCrory, eds.) 81-107, Proc. Sympos. Pure Math., 71, Amer. Math. Soc., Providence, RI, 2003.

[20] J. Etnyre, Legendrian and transversal knots, in 'Handbook of knot theory', (W. Menasco, M. Thistlethwaite, eds.) 105-185, Elsevier B. V., Amsterdam, 2005.

[21] D. Fuchs and D. Rutherford, Generating families and Legendrian contact homology in the standard contact space, J. Topol. 4(1) (2011), 190-226.

[22] H. Geiges, An introduction to contact topology, Cambridge Studies in Advanced Mathematics, 109, Cambridge University Press, Cambridge, 2008.

[23] A. Geng, Two surfaces in $D^{4}$ bounded by the same knot, J. Symplectic Geom. 9(2) (2011), 119-122.

[24] R. Golovko, A note on Lagrangian cobordisms between Legendrian submanifolds of $\mathbb{R}^{2 n+1}$, Pacific J. Math. 261(1) (2013), 101-116.

[25] R.E. Gompf, Symplectically aspherical manifolds with nontrivial $\pi_{2}$, Math. Res. Lett. 5(5) (1998), 599-603.

[26] J. Jordan and L. Traynor, Generating family invariants for Legendrian links of unknots, Algebr. Geom. Topol. 6 (2006), 895-933 (electronic).

[27] R. Lee and S. Weintraub, On the homology of double branched covers, Proc. Amer. Math. Soc. 123(4) (1995), 1263-1266.

[28] P. Lisca, On symplectic fillings of lens spaces, Trans. Amer. Math. Soc. 360(2) (2008), 765-799 (electronic).

[29] A. Loi and R. Piergallini, Compact Stein surfaces with boundary as branched covers of $B^{4}$, Invent. Math. 143(2) (2001), 325-348.

[30] D. McDuff, The structure of rational and ruled symplectic 4-manifolds, J. Amer. Math. Soc. 3(3) (1990), 679-712.

[31] D. McDuf, Symplectic manifolds with contact type boundaries, Invent. Math. 103(3) (1991), 651-671.

[32] P. Melvin and S. Shrestha, The nonuniqueness of Chekanov polynomials of Legendrian knots, Geom. Topol. 9 (2005), 1221-1252.

[33] L. Ng, Computable Legendrian invariants, Topology 42(1) (2003), 55-82.

[34] A. Oancea and C. Viterbo, On the topology of fillings of contact manifolds and applications, Comment. Math. Helv. 87(1) (2012), 41-69.

[35] H. Ohta and K. Ono, Symplectic fillings of the link of simple elliptic singularities, J. Reine Angew. Math. 565 (2003), 183-205.

[36] H. Ohta and K. Ono, Simple singularities and symplectic fillings, J. Differential Geom. 69(1) (2005), 1-42.

[37] H. Ohta and K. Ono, Examples of isolated surface singularities whose links have infinitely many symplectic fillings, J. Fixed Point Theory Appl. 3(1) (2008), 51-56.

[38] B. Ozbagci and A. Stipsicz, Contact 3-manifolds with infinitely many Stein fillings, Proc. Amer. Math. Soc. 132(5) (2004), 1549-1558 (electronic).

[39] L. Rudolph, Algebraic functions and closed braids, Topology 22(2) (1983), 191-202.

[40] L. Rudolph, Braided surfaces and Seifert ribbons for closed braids, Comment. Math. Helv. 58(1) (1983), 1-37.

[41] J. Sabloff and L. Traynor, Obstructions to Lagrangian cobordisms between Legendrian submanifolds, Algebr. Geom. Topol. 13 (2013), 2733-2797.

[42] S. Sivek, A bordered Chekanov-Eliashberg algebra, J. Topol. 4(1) (2011), 73-104.

[43] I. Smith, Torus fibrations on symplectic four-manifolds, Turkish J. Math. 25(1) (2001), 69-95.

[44] R.P. Stanley, Enumerative combinatorics, 1, Vol. 49 of Cambridge Studies in Advanced Mathematics, 2nd ed., Cambridge University Press, Cambridge, 2012, ISBN 978-1-107-60262-5.

[45] A. Stipsicz, Gauge theory and Stein fillings of certain 3-manifolds, Turkish J. Math. 26(1) (2002), 115-130.

[46] L. Traynor, Generating function polynomials for Legendrian links, Geom. Topol. 5 (2001), 719760 .

[47] C. Wendl, Strongly fillable contact manifolds and J-holomorphic foliations, Duke Math. J. 151(3) (2010), 337-384. 
Department of Mathematics, University of Pennsylvania, Philadelphia, PA 19104, USA

E-mail address: ccao@sas.upenn.edu

Department of Mathematics, University of California, Davis, Davis, CA 95616, USA

E-mail address: npgallup@ucdavis.edu

Department of Mathematics, Boston College, Chestnut Hill, Ma 02467, USA

E-mail address: kyle.hayden@bc.edu

Department of Mathematics and Statistics, Haverford College, Haverford, PA 19041, USA

E-mail address: jsabloff@haverford.edu 
